\title{
Estudo da Curva de Crescimento de Animais da Raça Guzerá e seus Cruzamentos Alimentados a Pasto, com e sem Suplementação. 2. Avaliação dos Parâmetros da Curva de Crescimento ${ }^{1}$
}

\author{
Luís Orlindo Tedeschi ${ }^{2}$, Celso Boin ${ }^{3}$, Romeu Fernandes Nardon ${ }^{4}$, Paulo Roberto Leme ${ }^{5}$
}

\begin{abstract}
RESUMO - Este trabalho analisou os parâmetros da função não-linear de Gompertz para descrever o crescimento de machos e fêmeas da raça Guzerá e seus cruzamentos (3/4Guzerá - 1/4Pardo Suíço, 1/2Nelore - 1/4Pardo Suíço - 1/4Guzerá, 1/2Pardo Suíço-1/2Guzerá, 1/2Chianina - 1/4Pardo Suíço - 1/4Guzerá, 1/2Caracú - 1/2Guzerá, 1/2Caracú - 1/4Pardo Suíço - 1/4Guzerá) submetidos a três níveis de suplementação (sem suplementação, suplementação durante o período seco e suplementação durante o ano todo), a fim de verificar as causas de variação nos parâmetros. Existiu interação positiva entre níveis de suplementação e grupo racial e entre grupo racial e sexo para peso adulto e taxa de maturidade. Notou-se também interação entre níveis de suplementação e sexo para a taxa de maturidade e para o parâmetro de integração. Observou-se efeito significativo apenas de grupo racial no parâmetro de integração. A suplementação apenas no período seco não alterou a estimativa do peso adulto. Quando os animais receberam suplementação mais energética, a taxa de maturidade foi aumentada. Os animais que receberam suplementação durante o ano todo apresentaram menor peso adulto e maior taxa de maturidade. As fêmeas prenhas obtiveram maior peso adulto e menor taxa de maturidade. Uma equação alométrica $\left(\mathrm{r}^{2}=0,68\right)$ foi derivada para predizer taxa de maturidade a partir do peso adulto.
\end{abstract}

Palavras-chave: funções não-lineares, gado de corte, peso adulto, taxa de maturidade

\section{Growth Curve Analysis of Guzera and Their Crossbreds Fed Under Grazing with or without Supplementation. 2. Evaluation of Growth Curve Parameters}

\begin{abstract}
This paper evaluated the parameters of Gompertz nonlinear function to describe Guzera male and female growth curve, and their crossbreds (3/4Guzera - 1/4Brown Swiss, 1/2Nellore - 1/4Brown Swiss - 1/4Guzera, 1/2Brown Swiss - 1/2Guzera, 1/2Chianina - 1/4Brown Swiss - 1/4Guzera, 1/2Caracu - 1/2Guzera, 1/2Caracu - 1/4Brown Swiss - 1/4Guzera) submitted to three levels of supplementation: without supplementation, supplementation only during the dry season, and year-round supplementation. There were significant interactions between supplementation and breed, and breed and sex on mature weight and maturing rate; supplementation and sex on maturing rate and integration parameter; and breed effect on integration parameter. The dry season supplementation did not change mature weight. Increasing the energy content of the supplement increased the maturing rate estimates. Supplementation during year-round decreased the estimates of mature weight but increased maturing rate values. Calving status had a significant effect on mature weight and maturing rate estimates. An allometric equation with an $\mathrm{r}^{2}$ of 0.68 was derived to predict maturing rate from mature weight estimates.
\end{abstract}

Key Words: beef cattle, nonlinear function, mature weight, maturing rate

\section{Introdução}

Têm sido questionado vários efeitos da suplementação no crescimento de bovinos, principalmente no período de menor disponibilidade de forragens, com relação ao crescimento, à composição corporal e aos aspectos econômicos (EUCLIDES FILHO et al., 1997).

Sabe-se que animais que passaram por restrição alimentar podem recuperar o mesmo peso em relação aos animais que não sofreram restrição alimentar, todavia, o mesmo peso será alcançado em idade mais avançada. Esse efeito é chamado de crescimento ou ganho compensatório, podendo ser influenciado pela idade do animal, severidade e duração da restrição (RYAN, 1990).

O tamanho adulto pode ser modificado pelo manejo de alimentação. Quando ocorre restrição acentuada do nascimento até os 4 a 5 meses seguintes, o tamanho adulto do animal pode ser diminuído (RYAN, 1990). De forma contrária, elevado consu-

\footnotetext{
1 Parte da Tese de Mestrado apresentada à ESALQ/USP pelo primeiro autor. Parte do Projeto do Instituto de Zootecnia, SP, protocolo IZ-14.012/78.

2 Estudante de PhD, Cornell University, Ithaca, NY. Bolsista do CNPq. E.mail: Iot1@cornell.edu

3 Professor Aposentado do Depto. de Zootecnia/ESALQ/USP. E.mail: celsoboin@picture.com.br

4 Instituto de Zootecnia - Nova Odessa. E.mail: nardon@izsp.br

5 Professor do Depto. de Zootecnia/FZEA/USP. E.mail: prleme@usp.br
} 
mo, principalmente à base de concentrado, também pode diminuir o tamanho adulto, devido principalmente ao efeito da "constância da composição corporal", de forma que animais superalimentados atingem rapidamente a composição corporal adulta e "cessam" o seu crescimento normal, mesmo sem terem alcançado o seu tamanho adulto padrão (OWENS et al., 1995).

Além dos efeitos nutricionais, os efeitos genéticos parecem alterar de forma significativa o peso adulto dos animais sem modificar consideravelmente a sua taxa de maturação. Resultados que suportam essa relação foram discutidos por PEROTTO et al. (1997) com animais das raças com Holandês, Gir e Guzerá.

Fêmeas de reposição com altas taxas de maturidade (crescimento precoce) apresentam maior custo à mesma idade para animais com pesos adultos similares. Entretanto, quando comparadas ao mesmo peso, as fêmeas com altas taxas de maturidade possuem menores custos e, no geral, são mais eficientes energeticamente do que aquelas com menores taxas de maturidade, tanto no âmbito reprodutivo/ crescimento quanto no econômico (BROWN e BROWN, 1972). Da mesma forma, fêmeas com mesma taxa de maturidade, porém menor peso adulto, são mais eficientes economicamente.

O objetivo deste estudo foi verificar os efeitos das estratégias de suplementação nos parâmetros da curva de crescimento de Gompertz para a raça Guzerá e seus cruzamentos com Nelore, Chianina, Caracú e Pardo Suíço. A seleção da curva de crescimento de Gompertz foi analisada por TEDESCHI et al. (2000) para o mesmo conjunto de dados apresentados neste trabalho.

\section{Material e Métodos}

Este estudo analisou o conjunto de dados obtidos pelo projeto IZ-14.012/78 - "Pastejo contínuo pósdesmama com e sem suplementação protéica nas secas", da Divisão de Bovinos de Corte do Instituto de Zootecnia/CPA/SAA.

Este projeto foi desenvolvido em dois anos consecutivos de nascimento (1977 e 1978, respectivamente experimentos 1 e 2) de machos e fêmeas para a coleta de peso vivo durante três a quatro anos. Esses animais são o resultado do cruzamento de fêmeas Guzerá (GU) com Pardo Suíço (PS), Nelore (NE), Chianina $(\mathrm{CH})$ e Caracú $(\mathrm{CA})$. A composição genética dos animais para os dois anos foi a seguinte: $\mathrm{GU}$ apenas, 3/4GU 1/4PS, 1/2NE 1/4PS 1/4GU, 1/2PS
1/2GU, 1/2CH 1/4PS 1/4GU, 1/2CA 1/2GU e 1/2CA 1/4PS 1/4GU. Os níveis de suplementação avaliados foram: sem suplementação (S1), suplementação apenas durante o período seco do ano (S2) e suplemetação durante o ano todo (S3). A suplementação durante o período seco (S2) teve duração média de 150 dias.

Para os animais do experimento 1 (nascidos em 1977 e suplementados em 1978), os grupos raciais GU, 3/4GU 1/4PS, 1/2NE 1/4PS 1/4GU, 1/2PS $1 / 2 \mathrm{GU}$ e $1 / 2 \mathrm{CH} 1 / 4 \mathrm{PS} 1 / 4 \mathrm{GU}$ foram submetidos aos seguintes níveis de suplementação: sem (S1) e com suplementação "nas secas" (S2) de 0,5 kg de farelo de soja por animal por dia. O total de animais utilizados nessa análise foi de 61 machos e 58 fêmeas. Este experimento foi designado de ano base 1978 , pois a suplementação teve início nesse ano.

Para os animais do experimento 2 (nascidos em 1978 e suplementados em 1979), todos os grupos raciais foram avaliados sob os níveis de suplementação sem (S1) e com suplementação apenas durante o período "das secas" (S2). Apenas os grupos raciais GU, 3/4GU 1/4PS e 1/2NE 1/4PS 1/4GU receberam suplementação total (S3). O suplemento foi constituído de $69,1 \%$ de farelo de trigo, $29,6 \%$ de milho e $1,3 \%$ de uréia (base seca) e fornecido $1,5 \mathrm{~kg}$ por animal por dia. O total de animais utilizados nesta análise foi de 90 machos e 89 fêmeas. Este experimento foi designado de ano base 1979 .

Os animais foram manejados em pastagem de colonião em sistema de pastejo rotacionado na Estação Experimental de Zootecnia de Andradina/SP e no Posto Experimental de Castilho/SP. A suplementação foi fornecida apenas durante o período matutino. A lotação média foi mantida ao redor de 2 animais/ha. Os machos foram castrados quando atingiram aproximadamente 1 ano de idade. Os animais de cada nível de suplementação permaneceram em pastos separados somente durante o período de suplementação. A fim de minimizar o efeito dos pastos no desempenho dos animais, foi efetuado o rodízio dos animais, procurando-se manter os diferentes níveis de suplementação em pastagens homogêneas (oferta de forragem semelhante). Machos e fêmeas permaneceram em pastos separados, com exceção do primeiro ano de suplementação. As pesagens foram efetuadas, a cada 28 dias, nos animais em jejum de 18 horas de água e alimento. Todos os animais foram periodicamente vacinados, vermifugados e banhados contra carrapatos.

Foi considerado ajuste de peso vivo em jejum para as fêmeas que se apresentavam prenhas. Dessa forma, 
1580 Rev. bras. zootec.

foram avaliados os machos e as fêmeas sem (F) e com $\left(F_{a j}\right)$ ajuste do peso vivo para os pesos do feto e dos tecidos anexos, das equações empíricas de peso total do útero, propostas por PRIOR e LASTER (1979).

As análises estatísticas foram efetuadas pelo software SAS® (1987) para cada ano (1 e 2). A análise de variância foi realizada por intermédio do procedimento General Linear Models (GLM) adotando o seguinte modelo estatístico fatorial (DEAN e VOSS, 1999):

$$
\begin{gathered}
Y_{i j k}=\mu+T_{i}+S_{j}+R_{k}+T_{i} * S_{j}+T_{i} * R_{k}+S_{j} * R_{k}+ \\
T_{i} * S_{j} * R_{k}+\varepsilon_{i j k}
\end{gathered}
$$

em que $\mathrm{Y}_{\mathrm{ijk}}$ é parâmetro da função de Gompertz; $\mu$, média geral; T, efeito de níveis de suplementação; $S$, feito de sexo; R, efeito do grupo racial; e $\varepsilon_{\mathrm{ijk}}$, erro aleatório não-controlável.

$\mathrm{Na}$ análise univariada, a comparação entre médias dos parâmetros de cada função foi realizada por meio do teste REGWF (Ryan-Einot-Gabriel-Welsh F; SAS, 1987), em nível de 5\%. Quando a interação foi significativa, cada fator foi avaliado dentro de cada níveis do outro fator e vice-versa (LITTELL et al., 1991), usando-se o mesmo teste estatístico.

O efeito de parição nos parâmetros da função de Gompertz também foi avaliado. Esse fator foi incluído no modelo estatístico citado acima bem como as suas interações. Os níveis de parição foram (P1) animais que não pariram, (P2) animais que pariram apenas com três anos de idade e (P3) animais que pariram, consecutivamente, com três e quatro anos de idade.

O procedimento NLIN do software SAS (SAS, 1987) foi utilizado com o método Gauss-Newton (HARTLEY, 1961; LAWTON et al., 1972) para a convergência dos dados à função de Gompertz conforme os procedimentos delineados por PEREIRA e ARRUDA (1987) e SAS (1987). A função de Gompertz foi previamente escolhida, por apresentar melhor convergência e descrição do crescimento desses animais (TEDESCHI et al., 2000).

\section{Resultados e Discussão}

\section{Análise do experimento 1}

As interações entre níveis de suplementação e grupo racial e entre sexo e grupo racial foram significativas $(\mathrm{P}<0,05)$ respectivamente para os parâmetros " $A$ " e " $b$ ”, e " $A$ "e “ $k$ ".

Constata-se, na Tabela 1, que os animais GU apresentaram menor estimativa de peso adulto ( “ $A$ ”), quando foram suplementados $(\mathrm{P}<0,05)$ e os
3/4GU 1/4PS, menor estimativa de peso adulto, quando não foram suplementados. Não houve diferença para a estimativa de peso adulto para os demais grupos raciais $(\mathrm{P}>0,05)$. Estes dados sugerem que os animais sem suplementação apresentaram crescimento compensatório (RYAN, 1990), com exceção dos cruzados 3/4GU 1/4PS. Os dados do trabalho conduzido por VERA (1991) suportam essa hipótese de crescimento, pois os valores de peso adulto de fêmeas Brahman encontrados sob regime de restrição alimentar, em pastagem, não ultrapassaram $350 \mathrm{~kg}$, enquanto os valores estimados de peso adulto para esta raça estão ao redor de $500 \mathrm{~kg}$, em condições normais de crescimento, segundo DOREN et al. (1989). Para o parâmetro de integração (“b”), o efeito de suplementação foi significativo apenas para os animais 1/2PS $1 / 2 \mathrm{GU}(\mathrm{P}<0,05)$.

Quando os grupos raciais foram analisados dentro dos níveis de suplementação (Tabela 1), notou-se que os animais 3/4GU 1/4PS apresentavam menor peso adulto ( " $A$ ”) $(\mathrm{P}<0,05)$ em relação aos animais GU e 1/2NE 1/4PS 1/4GU, quando não foram suplementados (S1). Ao contrário deste resultado, no nível de suplementação "nas secas" (S2) os animais GU apresentaram menor peso adulto (" $A$ ”) em rela-

Tabela 1 - Estudo da interação entre níveis de suplementação e grupo racial para o peso adulto ("A", kg) e para o parâmetro de integração ("b", kg) para os animais do ano de 1978

Table 1 - Effect of interaction between treatment and genetic groups on mature weight ("A", $\mathrm{kg}$ ) and on integration parameter

\begin{tabular}{|c|c|c|c|c|}
\hline \multirow{3}{*}{$\begin{array}{l}\text { Grupo racial } \\
\text { Genetic group }\end{array}$} & \multicolumn{4}{|c|}{$\begin{array}{c}\text { Nível de suplementação } \\
\text { Supplementation level }\end{array}$} \\
\hline & \multicolumn{2}{|c|}{ S1 } & \multicolumn{2}{|c|}{ S2 } \\
\hline & "A" & "b" & "A" & "b" \\
\hline 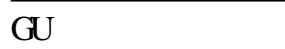 & $544^{\mathrm{Aa}}$ & $2,31^{\mathrm{Ab}}$ & $495^{\mathrm{Bb}}$ & $2,34^{\mathrm{Aa}}$ \\
\hline 3/4GU 1/4PS & $459^{\mathrm{Bb}}$ & $2,12^{\mathrm{Ac}}$ & $535^{\mathrm{Aa}}$ & $2,15^{\mathrm{Abc}}$ \\
\hline 1/2NE 1/4PS $1 / 4 \mathrm{GU}$ & $565^{\mathrm{Aa}}$ & $2,20^{\mathrm{Abc}}$ & $535^{\mathrm{Aab}}$ & $2,18^{\mathrm{Abc}}$ \\
\hline $1 / 2 \mathrm{PS} 1 / 2 \mathrm{GU}$ & $526^{\mathrm{Aab}}$ & $2,75^{\mathrm{Ba}}$ & $529^{\mathrm{Aab}}$ & $2,30^{\mathrm{Aab}}$ \\
\hline 1/2CH1/4PS $1 / 4 \mathrm{GU}$ & $477^{\mathrm{Aab}}$ & $2,24^{\mathrm{Abc}}$ & $510^{\mathrm{Aab}}$ & $2,04^{\mathrm{Ac}}$ \\
\hline
\end{tabular}
("b", kg) for the experiment based on 1978 data

Letras distintas indicam diferença estatística pelo teste REGWF, a $5 \%$, sendo que as letras maiúsculas estão associadas aos níveis de suplementação dentro de grupos raciais (linhas) e as minúsculas, aos grupos raciais dentro de níveis de suplementação (colunas) do mesmo parâmetro. Os números indicam médias ajustadas pelo LSMeans. Níveis de suplementação são S1: sem suplementação e S2: com suplementação durante o período seco. Raças são GU: Guzerá, PS: Pardo Suíço, NE: Nelore e CH: Chianina.

Different letters indicate statistical difference at .05 by REGWF test. Capital letters indicate difference among treatments within genetic groups (rows) and small letters indicate difference among genetic groups within treatments (columns). Numbers indicate LSMeans adjusted mean. Supplementations are S1: without supplementation and S2: supplementation during dry season. Breeds are GU: Guzera, PS:Brown Swiss, NE: Nellore, and CH: Chianina. 
ção aos 3/4GU 1/4PS. Estes dados sugerem que os animais $3 / 4 \mathrm{GU} 1 / 4 \mathrm{PS}$ foram mais sensíveis à não suplementação do que os demais e os animais GU não responderam de forma similar aos demais cruzados, quando suplementados durante o perído "das secas". Para o parâmetro " $b$ ", o grupo racial 1/2PS $1 / 2 \mathrm{GU}$ mostrou maior valor $(\mathrm{P}<0,05)$ para o nível de suplementação $\mathrm{S} 1$, enquanto, para o nível de suplementação $S 2$, os grupos raciais GU e 1/2PS 1/2GU foram maiores $(\mathrm{P}<0,05)$. Os dados de LOXTON et al. (1992) mostraram que machos Brahman com suplementação protéica $(0,5$ a $1 \mathrm{~kg}$ de farelo de algodão), durante o período "das secas", obtiveram maior peso adulto ao abate do que animais sem suplementação. Entretanto, os autores não puderam identificar diferença entre a suplementação apenas no primeiro ano e apenas no segundo ano (LOXTON et al., 1992).

$\mathrm{Na}$ Tabela 2, constata-se que a estimativa de peso adulto para as fêmeas $\left(\mathrm{F}\right.$ e $\left.\mathrm{F}_{\mathrm{aj}}\right)$ foi menor $(\mathrm{P}<0,05) \mathrm{em}$ relação aos machos, com exceção do grupo racial 1/2CH 1/4PS 1/4GU (P>0,05). Esse fato pode estar relacionado ao consumo de matéria seca do pasto. A estimativa do peso adulto para as fêmeas não foi diferente entre grupos raciais $(\mathrm{P}>0,05)$, entretanto, os machos $1 / 2 \mathrm{PS} 1 / 2 \mathrm{GU}$ apresentaram maior peso adulto que os $1 / 2 \mathrm{CH} 1 / 4 \mathrm{PS} 1 / 4 \mathrm{GU}$ e GU $(\mathrm{P}<0,05)$. A Tabela 3 mostra que as fêmeas obtiveram maior $(\mathrm{P}<0,05)$ es-

Tabela 2 - Estudo da interação entre grupo racial e sexo para o peso adulto ("A", kg) para os animais do ano de 1978

Table 2 - Effect of interaction between genetic groups and sex on mature weight ("A", kg) for the experiment based on 1978 data

\begin{tabular}{lccc}
\hline $\begin{array}{l}\text { Grupo racial } \\
\text { Genetic group }\end{array}$ & \multicolumn{3}{c}{$\begin{array}{c}\text { Sexo } \\
\text { Sex }\end{array}$} \\
\cline { 2 - 4 } & $\mathrm{F}$ & $\mathrm{F}_{\mathrm{aj}}$ & $\mathrm{M}$ \\
\hline GU & $465^{\mathrm{Ba}}$ & $457^{\mathrm{Ba}}$ & $628^{\mathrm{Ab}}$ \\
$3 / 4 \mathrm{GU} 1 / 4 \mathrm{PS}$ & $431^{\mathrm{Ba}}$ & $420^{\mathrm{Ba}}$ & $658^{\mathrm{Aab}}$ \\
$1 / 2 \mathrm{NE} 1 / 4 \mathrm{PS} 1 / 4 \mathrm{GU}$ & $476^{\mathrm{Ba}}$ & $460^{\mathrm{Ba}}$ & $662^{\mathrm{Aab}}$ \\
$1 / 2 \mathrm{PS} \mathrm{1/2GU}$ & $404^{\mathrm{Ba}}$ & $396^{\mathrm{Ba}}$ & $732^{\mathrm{Aa}}$ \\
$1 / 2 \mathrm{CH} 1 / 4 \mathrm{PS} 1 / 4 \mathrm{GU}$ & $492^{\mathrm{Aa}}$ & $482^{\mathrm{Aa}}$ & $519^{\mathrm{Ac}}$
\end{tabular}

Letras distintas indicam diferença estatística pelo teste REGWF, a $5 \%$, sendo que as letras maiúsculas estão associadas aos sexos dentro de grupos raciais (linhas) e as minúsculas, aos grupos raciais dentro de sexos (colunas). Os números indicam médias ajustadas pelo LSMeans. Sexos são $F$ : fêmeas sem ajuste, $F_{a j}$ : fêmeas com ajuste de peso vivo e M: machos. Raças são GU: Guzerá, PS: Pardo Suíço, NE: Nelore e $\mathrm{CH}$ :Chianina.

Different letters indicate statistical difference at .05 by REGWF test. Capital letters indicate difference among sex within genetic groups (rows) and small letters indicate difference among genetic groups within sex (columns). Numbers indicate LSMeans adjusted mean. Sexes are F: females withoutbody weight adjustment for fetal tissues, $F_{a j}$ : females with body weight adjustment for fetal tissues, and M: males. Breeds are GU: Guzera, PS: Brown Swiss, $\mathrm{NE}$ : Nellore and $\mathrm{CH}$ : Chianina. timativa de taxa de maturidade ( “ $k$ ") em relação aos machos, com exceção dos animais 1/2CH 1/4PS $1 / 4 \mathrm{GU}$ que não diferiram entre sexos $(\mathrm{P}>0,05)$. A taxa de maturidade não diferiu entre grupos raciais para os machos $(\mathrm{P}>0,05)$, entretanto, para as fêmeas $1 / 2 \mathrm{PS}$ $1 / 2 \mathrm{GU}$, tanto com $\left(\mathrm{F}_{\mathrm{aj}}\right)$ ou sem $(\mathrm{F})$ ajuste de peso, o valor de " $k$ " foi maior $(\mathrm{P}<0,05)$ que os demais grupos raciais.

A relação inversa entre a estimativa de peso adulto e a taxa de maturidade é bastante conhecida na literatura (FITZHUGH, 1976), ou seja, animais que apresentam grande peso adulto geralmente crescem a uma taxa relativa menor que os animais de menor peso adulto, conforme citado em vários trabalhos (FREITAS et al., 1997; CARRIJO e MOURA, 1999).

Os resultados encontrados para este experimento (1978) mostram que a estimativa de peso adulto de animais GU e 3/4GU 1/4PS foi modificada pela suplementação "das secas". Os animais cruzados, quando suplementados nas "secas", tenderam a apresentar maior peso adulto, provavelmente, devido a outros fatores que não foram analisados, como por exemplo a quantidade de leite consumido. Houve diferença entre sexos para a estimativa da taxa de maturidade e do peso adulto entre os grupos raciais.

Análise do experimento 2

Este experimento não apresentou efeito de interação significativa, da mesma forma que o experi-

Tabela 3 - Estudo da interação entre grupo racial e sexo para a taxa de maturidade ( " $k$ ", \%) para os animais do ano de 1978

Table 3 - Effect of interaction between genetic groups and sex on maturing rate ("k", $\mathrm{kg}$ ) for the experiment based on 1978 data

\begin{tabular}{lccc}
\hline $\begin{array}{l}\text { Grupo racial } \\
\text { Genetic group }\end{array}$ & \multicolumn{3}{c}{$\begin{array}{c}\text { Sexo } \\
\text { Sex }\end{array}$} \\
\cline { 2 - 4 } & $\mathrm{F}$ & $\mathrm{F}_{\mathrm{aj}}$ & $\mathrm{M}$ \\
\hline GU & $0,25^{\mathrm{Ad}}$ & $0,25^{\mathrm{Ac}}$ & $0,20^{\mathrm{Ba}}$ \\
$3 / 4 \mathrm{GU} 1 / 4 \mathrm{PS}$ & $0,32^{\mathrm{Ab}}$ & $0,33^{\mathrm{Ab}}$ & $0,22^{\mathrm{Ba}}$ \\
$1 / 2 \mathrm{NE} 1 / 4 \mathrm{PS} 1 / 4 \mathrm{GU}$ & $0,31^{\mathrm{Abc}}$ & $0,32^{\mathrm{Ab}}$ & $0,23^{\mathrm{Ba}}$ \\
$1 / 2 \mathrm{PS} 1 / 2 \mathrm{GU}$ & $0,40^{\mathrm{Aa}}$ & $0,41^{\mathrm{Aa}}$ & $0,19^{\mathrm{Ba}}$ \\
$1 / 2 \mathrm{CH} 1 / 4 \mathrm{PS} 1 / 4 \mathrm{GU}$ & $0,25^{\mathrm{Acd}}$ & $0,25^{\mathrm{Ac}}$ & $0,22^{\mathrm{Aa}}$ \\
\hline
\end{tabular}

Letras distintas indicam diferença estatística pelo teste REGWF, a $5 \%$, sendo que as letras maiúsculas estão associadas aos sexos dentro de grupos raciais (linhas) e as minúsculas, aos grupos raciais dentro de sexos (colunas). Os números indicam médias ajustadas pelo LSMeans. Sexos são $F$ : fêmeas sem ajuste, $F_{\text {aj }}$ : fêmeas com ajuste de peso vivo e M: machos. Raças são GU: Guzerá, PS: Pardo Suíço, NE: Nelore e $\mathrm{CH}$ :Chianina.

Different letters indicate statistical difference at .05 by REGWF test. Capital letters indicate difference among sex within genetic groups (rows) and small letters indicate difference among genetic groups within sex (columns). Numbers indicate LSMeans adjusted mean. Sexes are F: females withoutbody weight adjustment for fetal tissues, $F_{\text {aj: }}$ females with body weight adjustment for fetal tissues, and M: males. Breeds are GU: Guzera, PS: Brown Swiss, $\mathrm{NE}$ : Nellore and $\mathrm{CH}$ : Chianina. 
1582 Rev. bras. zootec.

mento 1. Apenas os efeitos de sexo (para os parâmetros " $A$ ", " $b$ " e " $k$ "), grupo racial (para os parâmetros " $b$ ” e “ $k$ ") e níveis de suplementação (para o parâmetro " $k$ ") foram observados.

O efeito de sexo está mostrado na Tabela 4 para os três parâmetros da curva de crescimento. As fêmeas $F$ e $F_{a j}$, as quais não diferiram entre si $(\mathrm{P}>0,05)$, apresentaram menor estimativa de peso adulto ( $A$ "), maior estimativa de taxa de maturidade ( " $k$ ") e maior estimativa do parâmetro " $b$ " em relação aos machos $(\mathrm{P}<0,05)$. As fêmeas apresentaram peso adulto $25 \%$ menor que os machos, concordando com os valores adotados pelo CSIRO (COMMONWEALTH SCIENTIFIC AND INDUSTRIAL RESEARCH ORGANIZATION, 1990) que variam de 17 a 29\%. Um dos principais componentes para a diferença de peso adulto e taxa de maturidade entre machos e fêmeas é a composição corporal, sendo que as fêmeas geralmente apresentam maior porcentagem de gordura que os machos, quando comparados ao mesmo peso (ROBELIN, 1986, ROBELIN et al., 1989).

A análise para os grupos raciais consta da Tabela 5. Para a taxa de maturidade, os animais 1/2PS $1 / 2 \mathrm{GU}$ apresentaram os maiores valores que os $1 / 2 \mathrm{CH} 1 / 4 \mathrm{PS} 1 / 4 \mathrm{GU}(\mathrm{P}<0,05)$, enquanto os demais grupos raciais não diferiram entre si. Para o parâmetro de integração, os grupos raciais GU, $1 / 2 \mathrm{PS} 1 / 2 \mathrm{GU}$ e $1 / 2 \mathrm{CA} 1 / 2 \mathrm{GU}$ não diferiram entre si $(\mathrm{P}>0,05)$, mas apresentaram maiores valores que

Tabela 4 - Efeito de sexo sobre o peso adulto ("A", $\mathrm{kg}$ ), no parâmetro de integração (" $b$ ", kg) e na taxa de maturidade (" $k$ ", \%) para os animais do ano de 1979

Table 4 - Effect of sex on mature weight ("A", $\mathrm{kg}$ ), integration factor ("b", $\mathrm{kg}$ ), and on maturing rate (" $k$ ", \%) for the experiment based on 1979 data

\begin{tabular}{lrcr}
\hline Parâmetro & \multicolumn{3}{c}{ Sexo } \\
& \multicolumn{3}{c}{ Sex } \\
\cline { 2 - 4 } & $\mathrm{F}$ & $\mathrm{F}_{\text {aj }}$ & $\mathrm{M}$ \\
\hline "A" & $481^{\mathrm{B}}$ & $462^{\mathrm{B}}$ & $627^{\mathrm{A}}$ \\
" $b "$ & $2,34^{\mathrm{A}}$ & $2,30^{\mathrm{A}}$ & $2,10^{\mathrm{B}}$ \\
" $k "$ & $0,30^{\mathrm{A}}$ & $0,32^{\mathrm{A}}$ & $0,21^{\mathrm{B}}$ \\
\hline
\end{tabular}

Letras distintas na mesma linha indicam diferença estatística pelo teste REGWF, a $5 \%$. Os números indicam médias ajustadas pelo LSMeans. Sexos são F: fêmeas sem ajuste, $F_{a j}$ : fêmeas com ajuste de peso vivo e M: machos.

Different letters, within rows, indicate statistical difference at .05 by REGWF test. Numbers indicate LSMeans adjusted mean. Sexes are F: females without body weight adjustment for fetal tissues, $F_{a j}$ : females with body weight adjustment for fetal tissues and M: males. aos animais $3 / 4 \mathrm{GU} 1 / 2 \mathrm{PS}, 1 / 2 \mathrm{NE} 1 / 4 \mathrm{PS} 1 / 4 \mathrm{GU}$ e $1 / 2 \mathrm{CH} 1 / 4 \mathrm{PS} 1 / 4 \mathrm{GU}(\mathrm{P}<0,05)$.

Neste experimento, os animais que receberam suplementação no período "das secas" (S2) apresentaram maior taxa de maturidade que os animais não-suplementados $(\mathrm{S} 1)$, respectivamente, 0,0029 e $0,0027(\mathrm{P}<0,05)$.

\section{Suplementação durante o ano todo}

Nesta análise, as interações entre níveis de suplementação e sexo (para os parâmetros " $b$ " e “ $k$ ”) e níveis de suplementação e grupo racial (para o parâmetro " $k$ "), assim como os efeitos de sexo (para o peso adulto " $A$ ”) e grupo racial (para o parâmetro " $b$ ”), foram significativas $(\mathrm{P}<0,05)$.

Analisando-se o efeito de sexo dentro de níveis de suplementação (Tabela 6), observou-se que as fêmeas ( $F \quad$ e $F_{a j}$ ) que não receberam suplementação (S1) apresentaram maior valor para o parâmetro de integração em relação aos machos $(\mathrm{P}<0,05)$, ao passo que as fêmeas suplementadas durante o ano (S3) apresentaram maior valor para o parâmetro de integração em relação aos machos $(\mathrm{P}<0,05)$. Já os animais com suplementação durante o inverno (S2) não apresentaram diferença significativa entre sexos $(\mathrm{P}>0,05)$. Para as fêmeas $\left(\mathrm{F}\right.$ e $\mathrm{F}_{\mathrm{aj}}$ ), o nível de suplementação $\mathrm{S} 3$ aumentou o valor do parâmetro " $b$ " $(\mathrm{P}<0,05)$ enquanto para os machos não houve diferença estatística $(\mathrm{P}>0,05)$.

A Tabela 7 mostra que as fêmeas $\left(F\right.$ e $\left.F_{a j}\right)$

Tabela 5 - Efeito do grupo racial sobre o parâmetro de integração (" $b$ ", kg) e a taxa de maturidade ("k", $\%)$ para os animais do ano de 1979

Table 5 - Effect of genetic group on integration factor ("b", kg) and on Grupo racial maturing rate (" $k$ ", \%) for the experiment based on 1979 data

Genetic group

\begin{tabular}{lll}
\cline { 2 - 3 } & \multicolumn{1}{c}{$b^{\prime}$} & " $k "$ \\
\hline GU & $2,44^{\mathrm{a}}$ & $0,26^{\mathrm{bc}}$ \\
3/4GU 1/4PS & $2,13^{\mathrm{bc}}$ & $0,27^{\mathrm{bc}}$ \\
$1 / 2 \mathrm{NE} 1 / 4 \mathrm{PS} 1 / 2 \mathrm{GU}$ & $2,13^{\mathrm{bc}}$ & $0,27^{\mathrm{bc}}$ \\
$1 / 2 \mathrm{PS} 1 / 2 \mathrm{GU}$ & $2,55^{\mathrm{a}}$ & $0,36^{\mathrm{a}}$ \\
$1 / 2 \mathrm{CH} 1 / 4 \mathrm{PS} 1 / 4 \mathrm{GU}$ & $2,06^{\mathrm{c}}$ & $0,24^{\mathrm{c}}$ \\
$1 / 2 \mathrm{CA} 1 / 2 \mathrm{GU}$ & $2,49^{\mathrm{a}}$ & $0,31^{\mathrm{ab}}$ \\
$1 / 2 \mathrm{CA} 1 / 4 \mathrm{PS} 1 / 4 \mathrm{GU}$ & $2,25^{\mathrm{b}}$ & $0,28^{\mathrm{bc}}$ \\
\hline
\end{tabular}

Letras distintas na mesma coluna indicam diferença estatística pelo teste REGWF, a $5 \%$. Os números indicam médias ajustadas pelo LSMeans. Raças são GU: Guzerá, PS: Pardo Suíço, NE: Nelore, $\mathrm{CH}:$ Chianina e CA: Caracú.

Different letters, within column, indicate statistical difference at .05 by the REGWFtest. Numbers indicate LSMeans adjusted mean. Breeds are GU: Guzera, PS: Brown Swiss, NE: Nellore, CH: Chianina and CA: Caracu. 
apresentaram maior taxa de maturidade que os machos $(\mathrm{P}<0,05)$ para todos os níveis de suplementação e o nível de suplementação S3 (suplementação durante o ano todo) aumentou as taxa de maturidade apenas para as fêmeas $\left(F\right.$ e $\left.F_{a j}\right)$ $(\mathrm{P}<0,05)$, enquanto, para os machos, o nível de suplementação $\mathrm{S} 2$ não diferiu do $\mathrm{S} 3(\mathrm{P}>0,05)$, mas $\mathrm{S} 2$ e S3 diferiram do $\mathrm{S} 1 \quad(\mathrm{P}<0,05)$. Estes resultados indicam que a suplementação total tendeu a diminuir o tempo para as fêmeas atingirem o mesmo peso vivo em relação às femeas sem e com suplementação durante o inverno. Para os machos, apenas a suplementação de inverno foi suficiente para diminuir esse tempo de crescimento. Os resultados de fêmeas concordam com o estudo de WILLMS et al. (1998), que observaram aumento no peso vivo de vacas Hereford suplementadas a pasto durante 90 dias/ano por 3 anos consecutivos. Resultados similares também foram relatados por CHARMLEY et al. (1999).

Houve efeito significativo da interação entre níveis de suplementação e grupo racial para a taxa de maturidade. Observa-se, na Tabela 8, que, quando os animais foram suplementados apenas durante o inverno, não houve diferença na taxa de maturidade ( " $k$ ”) entre grupos raciais, mas, quando suplementados

Tabela 6 - Estudo da interação entre sexo e níveis de suplementação para o parâmetro de integração ( "b", kg) apenas para os animais do ano de 1979 que receberam suplementação durante todo ano

Table 6 - Effect of interaction between sex and treatment on integration factor ("b", kg) for animals with yearround supplementation from the experiment based on 1979 data

\begin{tabular}{lccc}
\hline \multirow{2}{*}{$\begin{array}{l}\text { Nível de supplementação } \\
\text { Suptentation level }\end{array}$} & \multicolumn{3}{c}{$\begin{array}{c}\text { Sexo } \\
\text { Sex }\end{array}$} \\
\cline { 2 - 4 } & $\mathrm{F}$ & $\mathrm{F}_{\mathrm{aj}}$ & $\mathrm{M}$ \\
\hline S1 & $2,34^{\mathrm{Ab}}$ & $2,32^{\mathrm{Ab}}$ & $2,03^{\mathrm{Ba}}$ \\
S2 & $2,22^{\mathrm{Ab}}$ & $2,20^{\mathrm{Ab}}$ & $2,12^{\mathrm{Aa}}$ \\
S3 & $3,16^{\mathrm{Aa}}$ & $3,16^{\mathrm{Aa}}$ & $2,23^{\mathrm{Ba}}$ \\
\hline
\end{tabular}

Letras distintas indicam diferença estatística pelo teste REGWF, a $5 \%$, sendo que as letras maiúsculas estão associadas aos sexos dentro de níveis de suplementação (linhas) e as minúsculas, aos níveis de suplementação dentro de sexos (colunas). Os números indicam médias ajustadas pelo LSMeans. Sexos são F: fêmeas sem ajuste, $F_{a:}$ : fêmeas com ajuste de peso vivo e $\mathrm{M}$ : machos. Níveis de suplementação são $\mathrm{S} 1$ : sem suplementação, $\mathrm{S} 2$ : com suplementação durante o período seco e S3: suplementação duranto o ano todo. Different letters indicate statistical difference at .05 by REGWF test. Capital letters indicate difference among sex within treatment (rows) and small letters indicate difference among treatment within sex (columns). Numbers indicate LSMeans adjusted mean. Sexes are F: females without body weight adjustment for fetal tissues, $F_{a j}$ : females with body weight adjustment for fetal tissues, and M:males. Supplementations are S1: withoutsupplementation, S2: supplementation during dry season, and S3: supplementation year-round. durante o ano todo, o grupo racial 3/4GU 1/4PS apresentou maior taxa de maturidade em relação aos animais GU $(\mathrm{P}<0,05)$, sugerindo melhor conversão alimentar, em decorrência do suplemento. Quando não foram suplementados, os animais 1/2NE 14PS 1/4GU apresentaram maior taxa de maturidade em relação ao 3/4GU 1/4PS $(\mathrm{P}<0,05)$. De forma geral, os animais que foram suplementados durante o ano apresentaram maior taxa de maturidade dentro de cada grupo racial $(\mathrm{P}<0,05)$.

$\mathrm{O}$ efeito de sexo para a estimativa de peso adulto mostrou que as fêmeas $F(459)$ e $F_{\text {aj }}$ (441) não diferiram entre si $(\mathrm{P}>0,05)$, mas diferiram dos machos $(607 ; \mathrm{P}<0,05)$. O parâmetro de integração ( " $b$ ") foi afetado pelo efeito de raças de forma que o valor para os animais $\operatorname{GU}(2,64)$ foi maior $(\mathrm{P}<0,05)$ que os animais $3 / 4 \mathrm{GU} 1 / 4 \mathrm{PS}$ $(2,35)$ e $1 / 2 \mathrm{NE} 1 / 4 \mathrm{PS} 1 / 4 \mathrm{GU}(2,32)$, entretanto, esses dois últimos não diferiram entre si $(\mathrm{P}>0,05)$.

O nível de suplementação S2 não alterou significativamente o peso adulto dos animais em relação aos animais do nível de suplementação S1, mas aumentou a taxa de maturidade apenas nos animais que receberam a suplementação mais energética (Ano 2), indicando alta relação entre a taxa de

Tabela 7 - Estudo da interação entre sexo e níveis de suplementação para a taxa de maturidade ( " $k$ ", \%) apenas para os animais do ano de 1979 que receberam suplementação durante o ano todo

Table 7 - Effect of interaction between sex and treatment on maturing rate ("k", \%) for animals with year-round supplementation from the experiment based on 1979 data

\begin{tabular}{lccc}
\hline \multirow{2}{*}{$\begin{array}{l}\text { Nível de suplementação } \\
\text { Supplemtation level }\end{array}$} & \multicolumn{3}{c}{$\begin{array}{c}\text { Sexo } \\
\text { Sex }\end{array}$} \\
\cline { 2 - 4 } & $\mathrm{F}$ & $\mathrm{F}_{\mathrm{aj}}$ & $\mathrm{M}$ \\
\hline $\mathrm{S} 1$ & $0,30^{\mathrm{Ab}}$ & $0,31^{\mathrm{Ab}}$ & $0,19^{\mathrm{Bb}}$ \\
$\mathrm{S} 2$ & $0,30^{\mathrm{Ab}}$ & $0,31^{\mathrm{Ab}}$ & $0,24^{\mathrm{Ba}}$ \\
$\mathrm{S} 3$ & $0,44^{\mathrm{Aa}}$ & $0,46^{\mathrm{Aa}}$ & $0,27^{\mathrm{Ba}}$ \\
\hline
\end{tabular}

Letras distintas indicam diferença estatística através do teste REGWF ao nível de $5 \%$, sendo que as letras maiúsculas estão associadas aos sexos dentro de níveis de suplementação (linhas) e as minúsculas aos níveis de suplementação dentro de sexos (colunas). Os números indicam médias ajustadas pelo LSMeans. Sexos são F: fêmeas sem ajuste, $F_{a j}$ : fêmeas com ajuste de peso vivo e M: machos. Níveis de suplementação são $\mathrm{S} 1$ : sem suplementação, S2: com suplementação durante o período seco e S3: suplementação duranto o ano todo. Distinct letters indicate statistical difference at .05 by the REGWF test. Capital letters indicate difference among sex within treatment (rows) and small letters indicate difference among treatment within sex (columns). Numbers indicate LSMeans adjusted mean. Sexes are F: females without body weight adjustment for fetal tissues, $F_{a j}$ : females with body weight adjustment for fetal tissues, and M:males. Supplementations are S1: withoutsupplementation, S2: supplementation during dry season, and S3: supplementation year-round. 
1584 Rev. bras. zootec.

Tabela 8 - Estudo da interação entre grupo racial e níveis de suplementação para a taxa de maturidade ( "k", \%) apenas para os animais do ano de 1979 que receberam suplementação total

Table 8 - Effect of interaction between genetic group and treatment on maturing rate ("k", $\mathrm{kg}$ ) for animals with year-round supplementation from the experiment based on 1979 data

\begin{tabular}{lllc}
\hline $\begin{array}{l}\text { Nível de suplementação } \\
\text { Supplementation level }\end{array}$ & \multicolumn{3}{c}{$\begin{array}{c}\text { Grupo racial } \\
\text { Genetic group }\end{array}$} \\
\cline { 2 - 4 } & GU & $3 / 4 \mathrm{GU} 1 / 4 \mathrm{PS}$ & $\begin{array}{c}1 / 2 \mathrm{NE} 1 / 4 \mathrm{PS} \\
1 / 4 \mathrm{GU}\end{array}$ \\
\hline S1 & $0,26^{\mathrm{ABb}}$ & $0,25^{\mathrm{Bc}}$ & $0,28^{\mathrm{Ab}}$ \\
S2 & $0,27^{\mathrm{Ab}}$ & $0,30^{\mathrm{Ab}}$ & $0,27^{\mathrm{Ab}}$ \\
S3 & $0,36^{\mathrm{Ba}}$ & $0,42^{\mathrm{Aa}}$ & $0,37^{\mathrm{ABa}}$ \\
\hline
\end{tabular}

Letras distintas indicam diferença estatística pelo teste REGWF, a $5 \%$, sendo que as letras maiúsculas estão associadas aos grupos raciais dentro de níveis de suplementação (linhas) e as minúsculas, aos níveis de suplementação dentro de grupos raciais (colunas). Os números indicam médias ajustadas pelo LSMeans. Níveis de suplementação são $\mathrm{S1}$ : sem suplementação, S2: com suplementação durante o período seco e S3: suplementação duranto o ano todo. Raças são GU: Guzerá, PS: Pardo Suíço e NE: Nelore.

Different letters indicate statistical difference at .05 by REGWF test. Capital letters indicate difference among genetic group within treatment (rows) and small letters indicate difference among treatment within genetic group (columns). Numbers indicate LSMeans adjusted mean. Supplementations are S1: without supplementation, S2: supplementation during dry season, and S3: supplementation year-round. Breeds are GU: Guzera, PS:Brown Swiss and NE: Nellore.

maturidade e o manejo (ambiente), conforme encontrado por OLIVEIRA et al. (1994), em que a herdabilidade da taxa de maturidade foi de $8 \%$.

\section{Efeito de parição nos parâmetros da curva de crescimento}

$\mathrm{O}$ efeito de parição (P1, P2 e P3), tanto no peso adulto como na taxa de maturidade, mostrou-se altamente significativo $(\mathrm{P}<0,001)$ e, no parâmetro " $b$ ", foi significativo $(\mathrm{P}<0,01)$ para as fêmeas sem ajuste de peso $(\mathrm{F})$, bem como para as fêmeas com ajuste de peso $\left(\mathrm{F}_{\mathrm{aj}}\right)$. Conforme relatado por vários pesquisadores (MORROW et al., 1978; TORRE e RANKIN, 1978; McCURLEY et al., 1984; e TORRE et al., 1990), a parição influi nos parâmetros da função de crescimento.

Os animais que não pariram (P1) apresentaram peso adulto superior em relação aos animais $\mathrm{P} 2$ e P3 $\left(540,441\right.$ e $454 \mathrm{~kg}$ para F; 540, 423 e $435 \mathrm{~kg}_{\text {para F }}$; ; $\mathrm{P}<0,05)$, respectivamente. Estes dados são bastante semelhantes aos resultados obtidos por MORROW et al. (1978), estudando o efeito de parição nos parâmetros de crescimento de vacas Hereford. BELTRAN et al. (1991) também observaram que animais que pariram consecutivamente apresentaram menor estimativa de peso adulto.

O efeito dos níveis de suplementação na taxa de maturidade apresentou-se significativamente superior para os animais suplementados durante o ano (S3) em relação aos animais com suplementação apenas no inverno ou sem suplementação, 0,$44 ; 0,31 ;$ e $0,30 \%$ para $\mathrm{Fe} 0,46 ; 0,32$; e $0,3 \%$ para $\mathrm{F}_{\mathrm{aj}}$, respectivamente; $\mathrm{P}<0,05$. Para o fator de integração, os animais do nível de suplementação S3 apresentaram maior " $b$ " em relação aos do níveis de suplementação $\mathrm{S} 2$ e S1, 3,16; 2,26; e 2,35 para Fe 3,16; 2,22; e 2,31 para $\mathrm{F}_{\mathrm{aj}}$, respectivamente; $\mathrm{P}<0,05$.

Os animais do grupo racial 1/2PS 1/2GU apresentaram taxa de maturidade superior aos do grupo racial GU, 3/4GU 1/4PS e 1/2CH 1/4PS 1/4GU $(\mathrm{P}<0,05)$ para $\mathrm{F}$ e superiores aos grupos raciais $\mathrm{GU}$ e $1 / 2 \mathrm{CH} 1 /$ 4PS $1 / 4 \mathrm{GU}$ para $\mathrm{F}_{\mathrm{aj}}$. O grupo racial $1 / 2 \mathrm{CA} 1 / 2 \mathrm{GU}$ para F e 1/2PS 1/2GU, 1/2CA 1/2GU e GU para $F_{a j}$ apresentaram maior parâmetro " $b$ " que os animais do grupo racial 1/2CH 1/4PS $1 / 4 \mathrm{GU}(\mathrm{P}<0,05)$. O efeito de grupo racial na taxa de maturidade e no fator de integração estão sumariados na Tabela 9.

$\mathrm{O}$ efeito de parição na taxa de maturidade mostrou que os animais que não pariram (P1) apresentaram taxa de maturidade inferior em relação aos animais que pariram com três anos de idade (P2) ou três e quatro anos (P3) de idade, 0,$23 ; 0,34$; e $0,34 \%$ para Fe 0,$23 ; 0,35$; e $0,35 \%$ para $\mathrm{F}_{\mathrm{aj}}$, respectivamente; $\mathrm{P}<0,05$. Resultados similares foram encontrados por McLAREN et al. (1982), em que vacas Hereford que pariram consecutivamente apresentaram maior taxa

Tabela 9 - Efeito de grupo racial na taxa de maturidade ("k", \%) e no fator de integração ( " $b$ ", kg) em cada sexo para o modelo estatístico com efeito de parição

Table 9 - Effect of genetic group on maturing rate (" $k$ ", \%) and on integration factor ("b", $\mathrm{kg}$ ) for each sex group using the statistical model including calving status as a source of variation

\begin{tabular}{|c|c|c|c|c|}
\hline \multirow{2}{*}{$\begin{array}{l}\text { Grupo racial } \\
\text { Genetic group }\end{array}$} & \multicolumn{2}{|c|}{$\mathrm{F}$} & \multicolumn{2}{|c|}{$\mathrm{F}_{\mathrm{aj}}$} \\
\hline & “ $k ”$ & " $b "$ & " $k "$ & " $b "$ \\
\hline$\overline{\mathrm{GU}}$ & $0,28^{\mathrm{cd}}$ & $2,56^{\mathrm{ab}}$ & $0,29^{\mathrm{bc}}$ & $2,54^{\mathrm{a}}$ \\
\hline 3/4GU 1/4PS & $0,33^{b c}$ & $2,34^{b c}$ & $0,35^{\mathrm{abc}}$ & $2,32^{a b}$ \\
\hline 1/2NE 1/4PS $1 / 4 \mathrm{GU}$ & $0,33^{a b c}$ & $2,38^{a b c}$ & $0,35^{\mathrm{abc}}$ & $2,36^{\mathrm{ab}}$ \\
\hline 1/2PS $1 / 2 \mathrm{GU}$ & $0,41^{\mathrm{a}}$ & $2,65^{\mathrm{ab}}$ & $0,42^{\mathrm{a}}$ & $2,60^{\mathrm{a}}$ \\
\hline $1 / 2 \mathrm{CH} 1 / 4 \mathrm{PS} 1 / 4 \mathrm{GU}$ & $0,25^{\mathrm{d}}$ & $2,13^{\mathrm{c}}$ & $0,26^{\mathrm{c}}$ & $2,10^{\mathrm{b}}$ \\
\hline $1 / 2 \mathrm{CA} 1 / 2 \mathrm{GU}$ & $0,37^{\mathrm{ab}}$ & $2,68^{\mathrm{a}}$ & $0,38^{\mathrm{ab}}$ & $2,59^{\mathrm{a}}$ \\
\hline $1 / 2 \mathrm{CA} 1 / 4 \mathrm{PS} 1 / 4 \mathrm{GU}$ & $0,36^{a b c}$ & $2,47^{a b c}$ & $0,36^{\mathrm{ab}}$ & $2,40^{\mathrm{ab}}$ \\
\hline
\end{tabular}

Letras distintas na mesma coluna indicam diferença estatística pelo teste REGWF, a 5\%. Sexos são $F$ : fêmeas sem ajuste e $F_{a}$ : fêmeas com ajuste de peso vivo. Raças são GU: Guzerá, PS: Pardo Suíço, NE: Nelore, $\mathrm{CH}$ :Chianina e CA: Caracú.

Different letters, within column, indicate statistical difference of .05 by REGWF test. Sexes are F: females without body weight adjustment for fetal tissues and $F_{a j}$ females with body weight adjustmentforfetal tissues. Breeds are GU: Guzera, PS: Brown Swiss, NE: Nellore, CH: Chianina and CA: Caracu. 
de maturidade. Para o parâmetro " $b$ ”, os animais que não pariram apresentaram menor " $b$ ” em relação aos que pariram com três anos de idade ou três e quatro anos de idade, 2,22; 2,47; e 2,42 para F; e 2,22, 2,41 e 2,41 para $\mathrm{F}_{\mathrm{aj}}$, respectivamente; $\mathrm{P}<0,05$ ).

Quando foi analisado o efeito de grupo racial dentro de cada ano, notou-se que não houve diferença entre grupos raciais para o ano de 1978, embora as fêmeas $1 / 2 \mathrm{CH} 1 / 4 \mathrm{PS} 1 / 4 \mathrm{GU}$ tenham apresentado maior estimativa de peso adulto em relação às fêmeas GU, 1/2PS 1/2GU, 1/2CA 1/2GU e 1/2CA 1/4PS 1/4GU $(\mathrm{P}<0,05)$ para o ano de 1979.

Fêmeas que não apresentam parição no segundo ano de idade, geralmente, possuem maior peso adulto e menor taxa de maturidade (MORROW et al., 1978). Resultados de literatura têm mostrado que as fêmeas que não apresentam parição em estádios iniciais da vida reprodutiva sofrem alterações nas curvas de crescimento (MORROW et al., 1978; McCURLEY et al., 1984), ao passo que a falta de parição em estádios mais avançados parece não provocar alterações nos padrões de crescimento (TORRE e RANKIN, 1978; TORRE et al., 1990).

De forma geral, a época de pesagem e a performance reprodutiva dos animais devem ser definidas e consideradas na função, pois existem interações entre esses fatores e os parâmetros das funções de crescimento (FITZHUGH, 1967; MORROW et al., 1978; LUDWIG et al., 1981; e McLAREN et al., 1982).

Relação entre as estimativas de peso adulto e taxa de maturidade

A relação alométrica entre o parâmetro “ $k$ ” e o parâmetro “ $A$ ” apresenta relação não-linear inversa (FREITAS et al., 1997). TEDESCHI et al. (2000) verificaram que os parâmetros “ $A$ " e " $k$ " apresentaram alta correlação negativa, da ordem de $-0,77$. Esta relação está de acordo com valores encontrado por FITZHUGH (1976).

A regressão logarítmica entre " $k$ " e " $A$ ” para todos os dados analisados (Equação 1) mostrou ${ }^{2} \mathrm{de}$ $68 \%$ e os valores dos parâmetros $a$ e $b$ foram 0,795 e $-1,244$, respectivamente.

$$
\log (k)=a+b \cdot \log (A) \quad \text { [Equação 1] }
$$

A transformação da Equação 1 para uma equação alométrica resultou na Equação 2 abaixo:

$$
\mathrm{k}=\frac{6,2418}{\mathrm{~A}^{1,2435}} \quad \text { [Equação 2] }
$$

O CSIRO (1990) utiliza a equação descrita por TAYLOR (1968) para estimar a taxa de maturidade quando o peso adulto é fornecido. TAYLOR (1968) analisou várias espécies de aves e mamíferos e, em média, encontrou a seguinte relação entre " $k$ " $\mathrm{e}$ " $A$ " (Equação 3):

$\mathrm{k}=\frac{0,47}{\mathrm{~A}^{0,27}}$, para o tempo expresso em meses; ou $\mathrm{k}=\frac{0,015 \hat{6}}{\mathrm{~A}^{0,27}}$, para o tempo expresso em dias.

Quando se fixou o peso adulto dos animais para $\mathrm{A}^{0,27}$ e se ajustou a Equação 2, o valor do numerador obtido foi de 0,0157 , ou seja, bastante próximo ao valor citado por TAYLOR (1968).

A Figura 1 mostra as Equações 2 e 3 (TAYLOR, 1968) para estimar da taxa de maturidade pelo peso adulto. A equação de TAYLOR (1968) subestimou a taxa de maturidade para os animais com peso adulto inferior a $400 \mathrm{~kg}$ e superestimou, quando o peso adulto foi superior a $700 \mathrm{~kg}$.

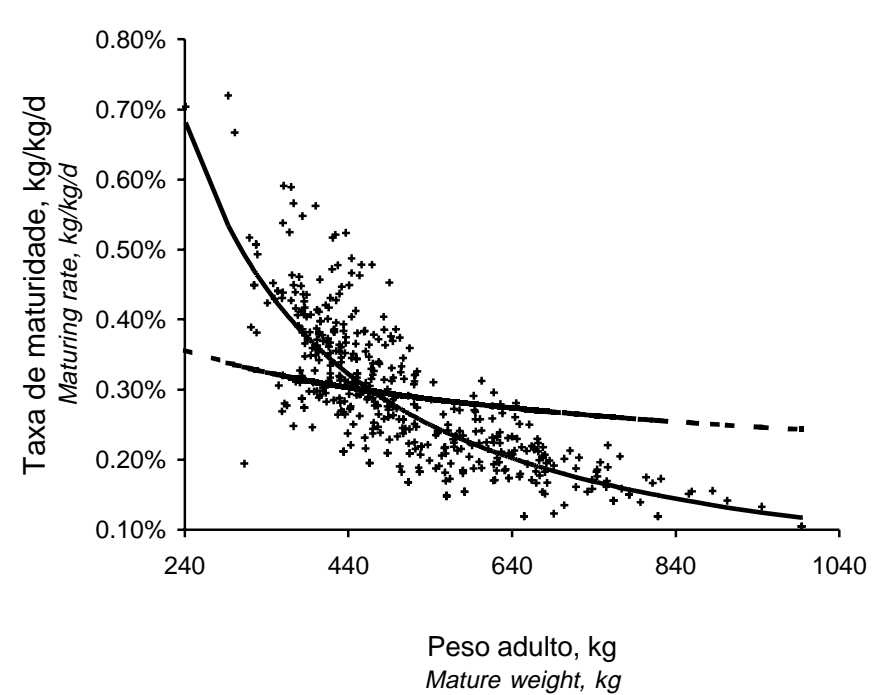

Figura 1 - Regressão entre o peso adulto ( $A$ ", kg) e a taxa de maturidade (" $k$ ", \%) para todos os animais analisados. Símbolos representam taxas de maturidade convergidas $(+)$, estimadas pela equação de Taylor (1968) (- - -) e estimadas pela equação $\mathrm{k}=6,242 /\left(\mathrm{A}^{1,2435}\right)(-)$.

Figure 1 - Relationship between mature weight ("A", kg) and maturing rate ("k", \%) for all animals evaluated. Symbols represent maturing rate converged (+), estimated by Taylor (1968) equation (- - ), and estimated by the equation $k=6.242$ / $\left(A^{1.2435}\right)(-)$. 


\section{Conclusões}

A suplementação no período "das secas" não modificou o peso adulto dos animais, mas aumentou a taxa de maturidade apenas nos animais que receberam a suplementação mais energética. Os animais que receberam suplementação durante o todo ano apresentaram menor peso adulto e maior taxa de maturidade em relação aos demais animais. Apenas os animais do grupo racial 3/4GU 1/4PS tenderam a apresentar alguma influência devido ao manejo de suplementação no peso adulto. A parição mostrou alta correlação com os parâmetros da função de crescimento, modificando o peso adulto. A regressão entre peso adulto e taxa de maturidade apresentou alta correlação $(r=0,82)$. Não houve diferença significativa entre as estimativas dos parâmetros das funções de crescimento para as fêmeas sem correção $(F)$ e com correção $\left(F_{a j}\right)$ do peso vivo.

\section{Agradecimento}

Aos auxiliares técnicos José Carlos M. Rasteiro, Mitsuharu Kuriama, Sebastião L. Camargo, Maria H. Camargo e Valfrido V. Lemos, aos campeiros Deraldo Manoel, Elízio Alves e Adércio Palomares e aos tratadores Pedro Daniel e Sebastião F. do Amaral, pela importante ajuda na condução deste experimento.

\section{Referências Bibliográficas}

BELTRAN, J.J., OLSON, T.A., ADAMS, E.L. Effect of reproductive history on estimating growth curve parameters in Angus cow. In: ANNUAL MEETING OF THE AMERICAN SOCIETY OF ANIMAL SCIENCE, 83, 1991, Forth Worth. J. Anim. Sci., 1991, p.5.

BROWN, C.J., BROWN, J.E. 1972. The influence of mature weight and rate of maturing on individual beef cow efficiency. Arkansas: University of Arkansas. 18p. (Bulletin, 774).

CARRIJO, S.M., MOURA, D.F.A. 1999. Description and comparison of growth parameters in Chianina and Nelore cattle breeds. Genetics and Molecular Biology, 22(2):187-196.

CHARMLEY, E., SMALL, J.A., McRAE, K.B. 1999. Influence of post-calving supplemental protein on calf performance and reproductive efficiency for beef cows fed silage. Can. J. Anim. Sci., 79(1):97-106.

COMMONWEALTH SCIENTIFIC AND INDUSTRIAL RESEARCH ORGANIZATION - CSIRO. 1990. Feeding standards for Australian livestock: ruminants. Melbourne: CSIRO. 266p.

DEAN, A., VOSS, D. 1999. Design and analysis of experiments. Springer, New York: Springer. 740p.

DOREN, P.E., BAKER, J.F., LONG, C.R. et al. 1989. Estimating parameters of growth curves of bulls. J. Anim. Sci., 67(6):14321445 .
EUCLIDES FILHO, K., EUCLIDES, V.P.B., FIGUEIREDO, G.R. et al. 1997. Efeito da suplementação com concentrado sobre a idade de abate e características de carcaça de bovinos Nelore. R. Bras. Zootec., 26(6):1096-1102.

FITZHUGH,H.A. 1976. Analysis of growth curves and strategies for altering their shapes. J. Anim. Sci., 42(4):1036-1051.

FREITAS, A.F., DURAES, M.C., TEIXEIRA, N.M. 1997. Growth curves of Holstein heifers managed in confinement. Arq. Bras. Med. Vet. Zootec., 49(1):85-93.

HARTLEY, H.O. 1961. The modified Gauss-Newton method for the fitting of nonlinear regression functions by least squares. Technometrics, 3(2):269-280.

LAWTON, W.H., SYLVESTRE, E.A., MAGGIO, S. 1972. Self modeling nonlinear regression. Techinometrics, 14(3):513-532.

LITTELL, R.C., FREUND, R.J., SPECTOR, P.C. 1991. SAS System for linear models. 3.ed. Cary, NC: SAS Institute Inc. 329p.

LOXTON, I.D., HOLROYD, R.G., BLIGHT, G.W. et al. Protein supplementation and growth enhancer strategies in successive years to maximise growth of Brahman cross steers grazing sown grass pastures on Brigalow lands. In: AUSTRALIAN SOCIETY OF ANIMAL PRODUCTION, 19, 1992, Rockbampton. Proceedings...Rockbampton, 1992, p.43-45.

LUDWIG, A., SILVA, M.A, OLIVEIRA, L.M. 1981. Ajustamento de modelos estatísticos exponenciais ao crescimento de gado Nelore. Pesq. Agropec. Bras., 39(3):383-387.

McCURLEY, J.R., BUTTS JR., W.T., BOVARD, K.P. 1984. Growth patterns of Angus, Hereford and Shorthorn cattle. I. Comparison of inbred and noningred lines, changes in patterns over time and effects of level of inbreeding and reproductive performance. J. Anim. Sci., 59(3):1194-1204.

McLAREN, J.B., MORROW, R.E.; BUTTS, W.T. 1982. Impact of numbers and frequency of weighings on bovine weight-age curve parameters. J. Anim. Sci., 54(1):51-57.

MORROW, R.E., McLAREN, J.B., BUTTS, W.T. 1978. Effect of age on estimates of bovine growth-curve parameters. $J$. Anim. Sci., 47(2):352-357.

OLIVEIRA, H.N., LOBO, R., PEREIRA, C.S. et al. Relationship among growth curve parameters, weights, and reproductive traits in Guzera beef cows. In: WORLD CONGRESS ON GENETICS APPLIED TO LIVESTOCK PRODUCTION, 5, 1994. Guelph. Proceedings... Guelph, 1994. p. 182-192.

OWENS,F.N., GILL,D.R., SECRIST,D.S. et al. 1995. Review of some aspects of growth and development of feedlot cattle. $J$. Anim. Sci., 73(10):3152-3172.

PEREIRA, A.R., ARRUDA, H.V. 1987. Ajuste prático de curvas na pesquisa biológica. Campinas: Fundação Cargill. 50p.

PEROTTO, D., CASTANHO, M.J.D.P., CUBAS, A.C. et al. 1997. Efeitos genéticos sobre as estimativas dos parâmetros das curvas de crescimento de fêmeas bovinas Gir, Guzerá, Holandês x Gir e Holandês x Guzerá. R. Bras. Zootec., 26(4):719-725.

PRIOR,R.L., LASTER,D.B. 1979. Development of the bovine fetus. J. Anim. Sci., 48(6):1546-1553.

ROBELIN, J. 1986. Growth of adipose tissues in cattle; partitioning between depots, chemical composition and cellularity. A review. Livest. Prod. Sci., 14(4):349-364.

ROBELIN, J., CHILLIARD, Y., AGABRIEL, J. Estimation of body lipids and proteins of Holstein Charolais and Limousine cows by dilution technique and adipose cell size. In: ENERGY METABOLISM OF FARM ANIMALS, 11, 1989, Lunteren. Proceedings... Pudoc, 1989. p.370-373.

RYAN, W.J. 1990. Compensatory growth in cattle and sheep. Nut. Abst. and Reviews, Series B, 60:653-664.

SAS INSTITUTE. 1987. SAS/STAT TM Guide for personal 
computers. 6.ed. Cary, NC: SAS Institute Inc. 1028p.

TAYLOR, C.S. 1968. Time taken to mature in relation to mature weight for sexes, strains and species of domesticated mamals and birds. Anim. Prod., 10(2):157-169.

TEDESCHI, L.O., BOIN, C., NARDON, R.F et al. 2000. Estudo da curva de crescimento da raça Guzerá e seus cruzamentos alimentados a pasto, com e sem suplementação. 1. Análise e seleção das funções não lineares. Rev. bras. zootec., 29(2):630-637.

TORRE, G.L., RANKIN, B.J. 1978. Factors affecting growth curve parameters of Hereford and Brangus cows. J. Anim. Sci., 46(3):604-613.

TORRE, G.L., CANDOTTI, J.J., VASCO, P. et al. Effect of growth curve parameters on cow efficiency. In: ANNUAL MEETING OF THE AMERICAN SOCIETY OF ANIMAL SCIENCE, 82, 1990, Little Rock. J. Anim. Sci., 1990, p.251. (Abstracts).
VERA,R.R. 1991. Growth and conception in continuously underfed Brahman heifers. Anim. Prod., 53(1):45-50.

WILLMS, W.D., RODE, L.M., FREEZE, B.S. 1998. Protein supplementation to enhance the performance of pregnant cows on rough fescue grasslands in winter. Can. J. Anim. Sci., 78(1):89-94.

Recebido em: 19/02/99

Aceito em: 11/04/00 